\title{
What is the role of healthcare managers in delivering safe care?
}

\section{A Hutchinson, P Barach}

\section{To what extent should healthcare boards be held accountable for clinical practice at the institutions they govern?}

W hen the State of New York Department of Health recently commissioned three quality improvement projects the topics seemed, at first sight, to be like many other quality projects before them. Each clinical topic is aimed at preventing recognised complications of interventions and each has an evidence base: perioperative use of beta blockers in non-cardiac surgery, prevention of thromboembolism, and surgical antimicrobial prophylaxis. Each group of hospitals that won the competition to run the projects has developed an evidence-based standard of care. Implementation of each clinical standard might be thought to run the risk that taking the evidence into practice may suffer from the usual barriers. ${ }^{1}$ This all seems routine, so what is new?

The differences, and the enhanced opportunities for success, lie in the context of the projects which are part of an initiative that aims to change practice and improve outcomes and, hence, improve safety. Each of the topics represents a type of adverse event regularly reported in the New York State Patient Occurrence Reporting and Tracking System (NYPORTS). ${ }^{2}$ Topic related preimplementation adverse event data are already available on the NYPORTS system for each participating hospital, and post-implementation data will be used to assess the effectiveness of the implementation package. These post-intervention data relate to important short term outcomes such as intraoperative or postoperative myocardial infarction, deep venous thrombosis or embolic events, and wound infections, together with associated mortality, morbidity, and costs.

Something else is different. Senior hospital executives are required to be part of the project team, which means that they-as well as the clinical team members-have explicit responsibility for delivering better and therefore safer care. All this raises intriguing questions about the accountability role of boards and management in delivering safe care. In the post Enron and WorldCom age, where boards are increasingly being encouraged to ask tough questions of the companies they govern, should healthcare boards be held accountable for clinical practice at the institutions they govern? Should boards be leading the charge to improve the safety and quality of patient care?

The involvement of chief executives, finance directors, and board members may be where patient safety programmes have the edge over "traditional" quality improvement programmes, for safety looks at quality from the other end of the telescope. Of course, every safety project should recognise the hard work of clinical teams who improve care by increments (sometimes over many years). But the safety perspective challenges the whole organisation. The safety approach says that those people who are not receiving evidence-based care represent a safety challenge. The people who are not receiving thromboembolism prophylaxis or who have had a myocardial infarction and been discharged from hospital without aspirin are at risk. Some will suffer an adverse event as a result of not receiving necessary advice or intervention.

"Safety looks at quality from the other end of the telescope"

Gaining the attention of hospital and primary care organisation chief executives and boards, sometimes for the first time, brings with it a recognition that quality of care is as much their responsibility as it is that of clinical teams. ${ }^{3}$ Leape $^{4}$ has referred to this as "reciprocal responsibility". Clinical teams take care to provide professional care under the circumstances in which they work. In turn, employers, managers and health service funding bodies have a responsibility to provide the circumstances, skills, people and equipment with which safe care can be delivered. This includes attention to the safety climate of the organisation, perhaps through what Weick and colleagues ${ }^{5}$ have characterised as the "process of mindful organising", in which there is a preoccupation with the likelihood of failure and a reluctance to simplify interpretations. This constant sense of unease might help to explain how some organizations are able to sustain high risk repeated encounters without suffering adverse events.
Safety brings with it a further dimension, one that many clinical teams have long struggled with. Safety is a systems issue. As knowledge advances and new technologies are deployed in the workplace, many undesired and unanticipated risks and consequences result. Although, disappointingly, some of the literature still refers to "medical error" and "human error", deep questioning related to adverse events will usually identify underlying systemic causes. ${ }^{67}$ Healthcare organisations are complex and are almost always under pressure, so the chance of failing to provide an element of care, or providing the wrong one, is always there if human activity is left unsupported by systems which can take up routine tasks, provide information, support decisions, and force safer functions.

Do support systems work? Evidence on guideline implementation suggests that there are no easy fixes when it comes to effecting change in clinical behaviour. ${ }^{7}$ But case studies on safety and system re-engineering from the Institute of Healthcare Improvement in the United States ${ }^{9}$ and from the National Health Service modernisation programme $^{10}$ have demonstrated real gains by engaging managers and by using systems approaches to quality and safety. Additionally, much of the research on improving the quality and safety of prescribing suggests that systematising the process can lead to substantial improvements. ${ }^{11}$

Quality used to be considered the preserve of clinical teams. Sometimes this was because of defensiveness and a wish to remain separate from management, sometimes because management was uninterested, more often because each side did not recognise each other's role. However, moving towards a culture of safety is at the heart of the ethical imperative of changing health care.

This places new requirements on healthcare organisations for even the introduction of clinical governance meant that healthcare organisations were more concerned with risk management than the more positive and encompassing concept of safety. Furthermore, every healthcare organisation faces significant safety and quality challenges that cannot all be fixed at once. Clinical and managerial partnership is required to set priorities and to support a culture of safe and effective practice.

The concept of safety as part of quality improvement enlarges the "quality envelope". Engaging senior management, funding agencies, and healthcare professionals with the safety agenda through a growing recognition of reciprocal responsibility and a focus on systems as well as people is the new dimension. It just might work.

Qual Saf Health Care 2003;12:161-162 


\section{Authors' affiliations \\ A Hutchinson, School of Health and Related Research (ScHARR), University of Sheffield, \\ Sheffield SI 4DA, UK \\ P Barach, Department of Anesthesia and \\ Critical Care, University of Chicago, Chicago, IL 60637, USA \\ Correspondence to: Professor A Hutchinson, School of Health and Related Research (ScHARR), Sheffield S1 4DA, UK; \\ allen.hutchinson@sheffield.ac.uk \\ REFERENCES \\ 1 Oxman AD, Thompson MA, Davis DA, et al. No magic bullets: a systematic review of}

interventions to improve professional practice. Can Med Assoc J 1995;153:1423-31.

2 New York State Patient Occurrence Reporting and Tracking System (NYPORTS). http://www.health.state.ny.us/ nysdoh/ (accessed 26 March 2003).

3 Mohr J, Abelson, H, Barach P. Leadership strategies in patient safety. J Qual Manage Health Care 2002;11:69-78.

4 Leape L. Reciprocal accountability: an overlooked dimension of safety. Alliance 2002; November, 24-6.

5 Weick KE, Sutcliffe KM, Obstfeld D. Organising for high reliability: processes of collective mindfulness. Res Organisational Behav 1999;21:81-123.

6 Woods DD, Cook RI. Nine steps to move forward from error. Cognition Technol Work 2002; 4: 137-44.
7 Dekker S. The field guide to human error investigations. Aldershot: Ashgate, 2002.

8 Grimshaw J, Russell IT. Effect of guidelines on medical practice: a systematic review of rigorous evaluations. Lancet 1993;342:1317-22.

9 Institute of Healthcare Improvement. htp://www.ihi.org/conferences/natforum/ handouts/M20_Chessare.pdf (accessed 1 April 2003).

10 National Health Service. htp://www.modernnhs.nhs.uk/scripts (accessed 1 April 2003).

11 Bates DW, Cohen M, Leape LL, et al. Reducing the frequency of errors in medicine using information technology. J Am Med Infor Assoc 2001;8:299-308.

\section{Aviation safety: a model for health care?}

\section{W Rutherford}

It is time to rethink the institutions and processes through which health care is delivered if a "culture of safety" is to be achieved.

"Patient safety" has become a prominent topic in the medical lexicon since the Institute of Medicine in 1999 released its landmark report "To Err Is Human". Much of the ensuing discussion surrounding an individual patient's well being treats "safety" as though it were a palpable, concrete entity which somehow can be created by command, manufacture, or spontaneous generation. Alas, it is neither so simple nor so tangible, nor is it accomplished by "doing what we always do, but doing it better". We must rethink the institutions and processes through which health care is offered.

If danger can be defined as the probability of incurring injury or death as a result of participating in, or being subjected to, a given activity or behavior, safety is the inverse-that is, the likelihood of emerging unharmed from the same behavior. In the first instance it is a relative term since life itself is a high risk phenomenon of finite duration. Life permits no absolute safety. By definition, patients are confronting some highlighted element of life's background risk when they enter themselves into the healthcare web. Those who seek care do so with the hope that they can find relief, all the while assuming that, in so doing, they do not expose themselves to new danger. Creating the environment where this assumption is justified is the challenge for patient safety activists.

In many potentially hazardous industries specific attitudes, processes, and procedures have been deployed actively to prevent induced harm, often quite aside and in addition to the purpose of the enterprise. We in health care give lip service to this moral imperative with the familiar admonition "first do no harm". How well do we respect it in our attitudes, processes and procedures? Do we know what we are doing?

Another contemporary industry exists which shares with health care some important attributes-high stakes; potentially lethal technology; primary participants who are strong willed, independent, and quick to slip into autocratic behavior; a history and tradition of deference to these key autocrats; work done in small, highly interdependent groups with little outside oversight or supervision. It has changed its behavior to enhance safety.

The airline industry has developed processes and disciplines with respect to safety (no favorable claims are made about this industry's business acumen) which can be instructive, even though not directly transplantable, to health care. Over the last 30 years three simultaneous and often interacting trends can be identified: (1) increasing system transparency (the threshold for reporting of untoward "events" has been lowered), (2) increasing standardization of procedures (the autonomy of the operator has been curtailed while preserving his authority), and (3) increasing efficiency in extracting value from all system elements-human, information and hardware-notions embodied in crew resource management (CRM). The result is a "culture of safety".

It is generally acknowledged that our health care does regularly violate this dictum. Resulting corrective efforts are often focused at the "sharp edge" of the health care process-the level of patient interface with the provider. This is understandable in our medical culture which subliminally suffuses students in their earliest days of medical school with the notion that "if I know enough, am smart enough, work hard enough, I will not make mistakes". However, profound adverse effects result from this misunderstanding of human behavior and human performance. They include strong psychological incentives-to add to legal ones in our "system" - for concealment, denial, and transfer of "blame" for the inevitable errors which do occur. Efforts focused at this sharp edge do not, and cannot, accomplish the command to do no harm; neither can a single soldier win a war nor an individual star constitute a basketball team. There are too many other forces and players involved. It is imperative that we look to the overall systems of delivering health care.

When we do, we find that there is no "system". Health care is delivered by a vast cottage industry which is populated by dispirited providers ${ }^{2}$ serving an increasingly disrespectful consumer, ${ }^{3}$ financed by reluctant governmental and private "third parties" and preyed upon by a politically well connected parade of plaintiffs' attorneys. A condition has been created wherein an "industry" supported by the most elegant new science and technology-and consuming great wealth-has grasped defeat from the jaws of victory. It seems to have lost its sense of purpose.

"Health care is delivered by a vast cottage industry populated by dispirited providers..."

It is impossible to hear physicians' discussions of similar cases and not be 
astounded (and appalled) by the practice they describe. The efficacy of so many therapeutic regimes is so doubtful as to cause many therapeutic choices to be made by ill supported opinion. There is a literature so confused and confusing that some common symptom constellations appear likely to receive about as many therapeutic interventions as there are practitioners consulted. Do all of them work? Do any of them work?

Very well defined evidence-based guidelines exist for treating several conditions-for example, asthma and hypertension. ${ }^{45}$ Yet surveys indicate that in fewer than $20 \%$ of patients is the treatment for these diseases meeting the standards of the guidelines. ${ }^{67}$

Well designed, carefully conducted clinical studies separate effective from ineffective regimens. Web based data collection could convert the existing diffuse practice into a powerful clinical study. Acceptable competing treatment protocols for common clinical conditions would be identified. Individual practitioners in this new practice environment could select one (as they often say they do) and apply it consistently. Providers could report results of their real-world interventions simultaneously with the medical record keeping and billing inputs in a well designed practice software suite. This could provide a good start towards identifying effective best practices. This has been the norm in treating pediatric leukemia patients for the last 20 years. ${ }^{8}$ Their results would enter the database along with others using the same and competing regimens. The large volume of data from an appropriately managed study would quickly offer guidance as to the most effective practices. We want evidence-based practice; let's generate good evidence.

The process of organizing and implementing such an initiative would eventually involve medical administration, education and practice at all levels, thus potentially restoring a sense of common purpose among diffuse and competing elements of the industry. Importantly, such a program could help bridge the expanding gap between the expertise in medical center ivory towers and the far distal branches of the healthcare apparatus.

There is no single notion-simple or grand-that will create a culture of safety for health care. No proclamations can fix the ills or grow a mature effective provider of care. But there are clues as to the direction in which we should start. Knowledge must be squeezed from the enterprise. Errors must be reported to permit engineering similar future errors out of a system. Treatment regimens must be validated or abandoned; effective ones must be delivered dependably to all who need them, even when that means public education to recruit those at silent risk.

Many conflicting pressures converge on health care. The industry seems to have neglected the potentials of a collective assertive pursuit of efficacy and safety in favor of a somewhat diffuse defence against these many assaults. The professions and their patient public could greatly benefit from initiatives that look toward new paradigms which realistically confront the problems patients bring to us, and the ones we bring to them.

Qual Saf Health Care 2003;12:162-163

\section{Author's affiliation}

W Rutherford, 9660 Wolf Road, Geneseo, IL 61254, USA; bill_rutherford@msn.com

\section{REFERENCES}

1 Kohn LT, Corrigan JM, Donaldson MS, eds. To err is human: building a safer health system. Institute of Medicine, Committee on Quality of Health Care in America. Washington, DC: National Academy Press, 1999.

2 Career Center for Employers. Hospitals and health networks. NEJM Career Center for Employers 2001;75 (www.nejmiobs.org/rpt, 2003).

3 Marcus LJ, Dorn BC. Renegotiating health care. AMNews 17 June 2002.

4 National Asthma Education and Prevention Program (NAEPP). Guidelines for the diagnosis and management of asthma. Expert Panel Report 2. NIH Publication No 97-4051. Bethesda, MD: National Institutes of Health, 1997 (updated 2002)

5 National Institutes of Health (NIH). The Sixth Report of the Joint National Committee on Prevention, Detection, Evaluation and Treatment of High Blood Pressure (JNC VI). $\mathrm{NIH}$ Publication No 98-4080. Bethesda, MD National Institutes of Health, 1998.

6 National Center for Health Statistics. Asthma prevalence, health care use and mortality 2000-2001. Hyattsville, MD: National Center for Health Statistics, US DHHS, Division of Data Services (http://www.cdc.gov/nchs/products/pubs/ pubd/hestats/asthma/asthma.htm, January 2003).

7 Meissner I, Whisnant JP, Sheps SG, et al. Detection and control of high blood pressure in the community: do we need a wake-up call? Hypertension 1999;34:466-71.

8 Simone JV, Lyons J. The evolution of cancer care for children and adults. J Clin Oncol 1998;16:2904-5. 